Research Paper

\title{
Generation and characterization of bound entanglement in optical qubits
}

\author{
Fumihiro KANEDA ${ }^{1}$, Ryosuke SHIMIZU ${ }^{2}$, Yasuyoshi MITSUMORI ${ }^{3}$, \\ Hideo KOSAKA ${ }^{4}$, and Keiichi EDAMATSU ${ }^{5}$ \\ 1,3,4,5 Research Institute of Electrical Communication, Tohoku University \\ ${ }^{2}$ PRESTO, Japan Science and Technology Agency (JST)
}

\begin{abstract}
For efficient quantum information processing (QIP), pure and strong entanglement in qubits has been thought to be indispensable. However, it was recently pointed out that bound entangled states, which involve undistillable entanglement between qubits, also have great potential for QIP. In this paper, we report the efficient generation of a four-qubit bound entangled state referred to as the Smolin state using photon polarization qubits. We observed the unlockable bound entanglement which is the specific property of the Smolin state.
\end{abstract}

\section{KEYWORDS}

Quantum information, entanglement, quantum state preparation

\section{Introduction}

Entanglement plays a crucial role in quantum information processing (QIP) [1]. It has been thought that pure and strongly entangled states are essential in QIP [2], [3]. In contrast, a class of the multipartite entanglement referred to as bound entanglement [4] has been considered the weak and useless entanglement for any QIPs, since the bound entanglement cannot be distilled into pure entanglement under local operations and classical communication (LOCC). However, it is interesting that the bound entanglement in a certain kind of bound entangled state can be activated when two of the parties coming together. The process is called "unlocking" [5]. It is also possible that two independent bound entangled states cooperatively distill the entanglement. The process is called "superactivation" [6]. These interesting properties of the bound entangled states have attracted attention in QIP applications, e.g., remote information concentration [7], secure QKD [8], and convertivility of multipartite pure entangled states [9].

Despite the theoretical interests, the experimental re-

Received October 31, 2010; Revised December 2, 2010; Accepted December 28, 2010.

${ }^{1)}$ kaneda@quantum.riec.tohoku.ac.jp, ${ }^{2)}$ r-simizu@pc.uec.ac.jp,

3)mitumori@riec.tohoku.ac.jp, ${ }^{4)}$ kosaka@riec.tohoku.ac.jp,

5) eda@riec.tohoku.ac.jp

DOI: $10.2201 / \mathrm{NiiPi} .2011 .8 .3$ alization of the bound entangled states is still a challenging task. To realize the bound entangled states using photons, we need (1) the high generation and detection efficiencies of multiple photon pairs, which decrease exponentially with increasing number of photons concerned, and (2) precise and stable control of the entanglement in multiple photon states, e.g., entanglement of the polarization states of the photons. The successful demonstrations of the four-qubit bound entangled state (Smolin state [5]) were reported recently by Amselem et al. [10] and by Lavoie et al. [11]. However, the generation rate of the Smolin state was not sufficiently high for further demonstration of the valuable QIP protocols based on bound entanglement. Thus, efficient generation of multi-qubit state is indispensable. In this paper, we report the generation and characterization of the four-qubit bound entangled Smolin state [5] encoded in photon polarization qubits, with much higher efficiency than ever reported.

\section{The bound-entangled Smolin state}

The Smolin state is an equal statistical mixture of pairs of the four Bell states. Its density matrix $\rho_{s}$ is 
given by

$$
\rho_{s}=\sum_{i=1}^{4}\left|\phi_{A B}^{i}\right\rangle\left\langle\phi_{A B}^{i}|\otimes| \phi_{C D}^{i}\right\rangle\left\langle\phi_{C D}^{i}\right|,
$$

where $\left|\phi^{i}\right\rangle \in\left\{\left|\phi^{ \pm}\right\rangle,\left|\psi^{ \pm}\right\rangle\right\}$are the two-qubit Bell states. The Bell states encoded in photon polarization are given by

$$
\begin{aligned}
& \left|\phi^{ \pm}\right\rangle=\frac{1}{\sqrt{2}}(|H H\rangle \pm|V V\rangle) \\
& \left|\psi^{ \pm}\right\rangle=\frac{1}{\sqrt{2}}(|H V\rangle \pm|V H\rangle) .
\end{aligned}
$$

Here, $|H\rangle$ and $|V\rangle$ indicate the single photon states having horizontal and vertical polarizations, respectively. As shown in Eq. (1), the Smolin state is separable across the bipartite cut $A B \mid C D$. Since the state has a symmetry with respect to the exchange of any two parties, it is separable with respect to any two-two bipartite cuts. This implies that no entanglement can be distilled between any two parties.

\section{Experiment}

To explore the multipartite entanglement including the bound entanglement, we have developed the experimental system optimized for efficient generation and fast evaluation of the multi-photon states [12]. The experimental setup for the generation and characterization of the Smolin state is sketched in Fig. 1. We used the third harmonics (wavelength $=343 \mathrm{~nm}$, average power $=200 \mathrm{~mW}$, pulse duration $=250 \mathrm{fs}$ ) of the mode-locked $\mathrm{Yb}$ laser (Amplitude Systems, t-Pulse 500) as a pump source for SPDC using two Type-II beta barium borate (BBO) crystals. The pump source has higher pulse energy $(\sim 60 \mathrm{~nJ})$ than those of typical multi-photon generation systems based on the second harmonics of TiSapphire lasers (10 20 nJ) (e.g., Ref. [13]). Moreover, the center wavelength $(686 \mathrm{~nm})$ of the SPDC pumped by our source is close to the spectral region where $\mathrm{Si}$ avalanche photodiodes (Si-APD) have maximum quantum efficiency $(\sim 65 \%)$. Each photon produced by the SPDC was passed through a band-pass filter (BPF, center wavelength $=686 \mathrm{~nm}$, FWHM $=1 \mathrm{~nm}$ ) for spectral filtering, and was led to a single-mode fiber (SMF) for spatial filtering. The filtering processes make photons indistinguishable except for their polarization. Each $\mathrm{BBO}$ crystal emits photon pairs having polarization entanglement in the Bell state $\left|\psi^{+}\right\rangle$. When the two BBO crystals simultaneously produce the pair of Bell states, the resulting four-photon state is given by

$$
\rho\left(\psi^{+}\right)=\left|\psi^{+}\right\rangle_{A B}\left\langle\psi^{+}|\otimes| \psi^{+}\right\rangle_{C D}\left\langle\psi^{+}\right| .
$$

The liquid crystal valuable retarder (LCVR) adds arbitrary phase shifts between two orthogonal polariza-

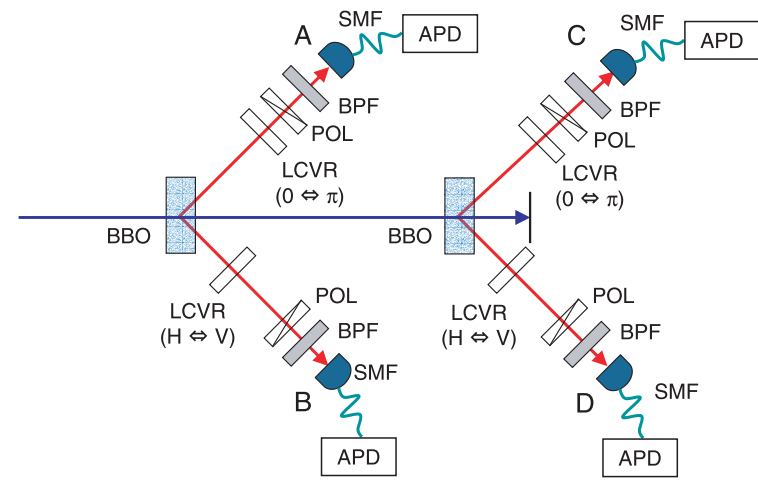

Fig. 1 Experimental setup for the generation of the Smolin state. LCVR: liquid crystal variable retarder, POL: polarizer, BPF: band-pass filter, SMF: single mode fiber, APD: avalanche photodiode detector.

tions; it can add $\pi$ phase shift between $\mathrm{H}$ and $\mathrm{V}$ polarizations, or, it can flip the two polarizations. In this way, the state $\left|\psi^{+}\right\rangle$generated by each BBO crystal can be converted into any of the four Bell states by using two LCVRs. Thus, by synchronously controlling the four LCVRs, we can prepare the Smolin state (1), i.e., random and uniform statistical mixture of the four states, $\rho\left(\psi^{+}\right), \rho\left(\psi^{-}\right), \rho\left(\phi^{+}\right)$, and $\rho\left(\phi^{-}\right)$. The retardation of each LCVR is set by a pseudo-random number generator operating at a rate of $3 \mathrm{~Hz}$. The polarizers, each of which consists of a quarter wave plate, a half wave plate and a polarization beam splitter, project the photons into any polarization states. The photons passed through the polarizers were detected by the single-photon avalanche photodiode detectors (APDs). Then, the signal pulses from the APDs are recorded by a multifold coincidence counter. The typical single, two-fold and four-fold coincidence rates were $1 \times 10^{5}, 2 \times 10^{4}$, and $4 \times 10^{1} \mathrm{sec}^{-1}$, respectively. To carry out the quantum state tomography, we recorded the four-fold coincidence signals for 256 combinations of the polarization projection measurements. The recording time for each projection measurement was four minutes, and the corresponding total measurement time was $\sim 18$ hours. For the tomography process, the maximally likelihood method [14] was used to avoid reconstruction of unphysical density matrices.

\section{Results and discussion}

Fig. 2 (a) and (b) show the real part of the reconstructed density matrix $\rho_{\text {exp }}$ and that of the Smolin state $\rho_{s}$, respectively. The fidelity $F=\left(\operatorname{Tr} \sqrt{\sqrt{\rho_{s}} \rho_{\exp } \sqrt{\rho_{s}}}\right)^{2}$ of the reconstructed matrix $\rho_{\text {exp }}$ to the ideal Smolin state $\rho_{s}$ was 0.876 . This value is comparable with those of 
a

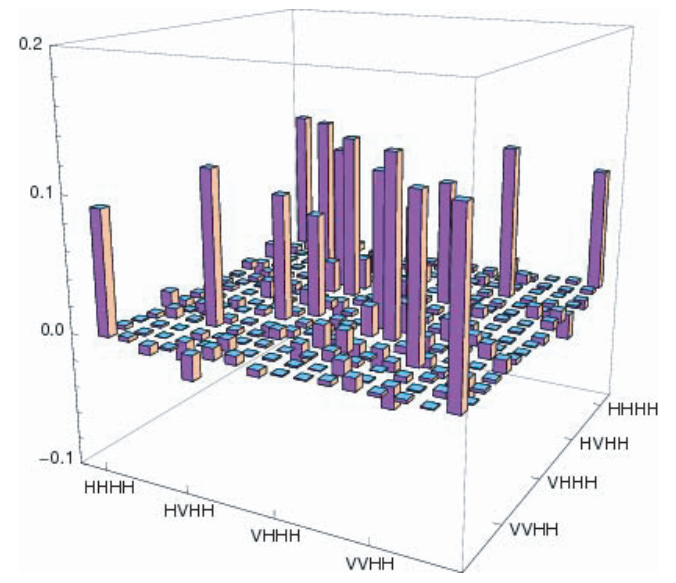

b

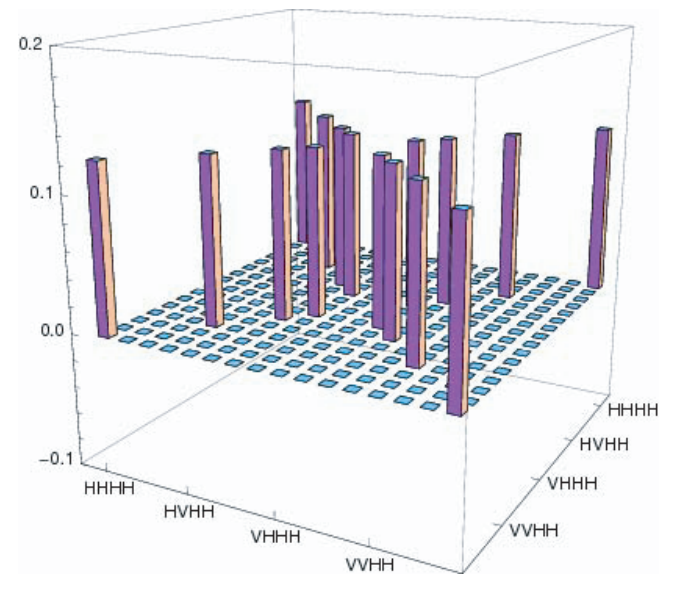

Fig. 2 Real part of the density matrices. (a) measured state $\rho_{\text {exp }}$ and (b) Smolin state $\rho_{s}$.

the previous experiments [10] of the Smolin state generation. It is noteworthy that the total measurement time of our experiment is almost 10 times shorter than those of the experiments ( $\sim 10$ days). This comes from the high multi-photon counting rate of our experimental system.

From the reconstructed density matrix $\rho_{\text {exp }}$, we evaluated the separability of the generated state across the bipartite cuts $\mathrm{AB}|\mathrm{CD}, \mathrm{AC}| \mathrm{BD}$, and $\mathrm{AD} \mid \mathrm{BC}$ in terms of the negativity [15], which quantifes the distillable entanglement of bipartite cuts under LOCC. The negativity of the density matrix which is composed of two subsystems $\rho_{a b}$ is given by

$$
N\left(\rho_{a b}, a\right)=\frac{\left\|\rho_{a b}^{T_{a}}\right\|-1}{2}
$$

Table 1 The negativity values for the two-two bipartite cuts.

\begin{tabular}{cccc}
\hline & $\rho_{\exp }$ & $\rho_{s}$ & $\rho\left(\psi^{+}\right)$ \\
\hline \hline$N(\rho, \mathrm{AB})$ & $0.06 \pm 0.01$ & 0 & 0 \\
$N(\rho, \mathrm{AC})$ & $0.11 \pm 0.02$ & 0 & 1.5 \\
$N(\rho, \mathrm{AD})$ & $0.12 \pm 0.02$ & 0 & 1.5 \\
\hline
\end{tabular}

where $\rho_{a b}^{T_{a}}$ represents the partial transpose with respect to the subsystem $a$, and $\left\|\rho_{a b}^{T_{a}}\right\|$ is the trace norm of $\rho_{a b}^{T_{a}}$. For instance, the separability across the bipartite cut $\mathrm{AB} \mid \mathrm{CD}$ is quantified as the $N\left(\rho_{\text {exp }}, \mathrm{AB}\right)$. The negativity values for the three bipartite cuts of the $\rho_{\text {exp }}$ are presented in Table 1, together with those of the Smolin state $\rho_{s}$ and the state $\rho\left(\psi^{+}\right)$in (3). The Smolin state $\rho_{s}$ has zero negativity for all the three bipartite cuts, while the state $\rho\left(\psi^{+}\right)$has finite values, i.e., finite distillable entanglement, for $\mathrm{AC} \mid \mathrm{BD}$ and $\mathrm{AD} \mid \mathrm{BC}$ cuts. For $\rho_{\text {exp }}$, the negativity values are all close to zero, indicating that it has almost no distillable entanglement as we expect for the Smolin state.

Furthermore, to quantify the entanglement without the one-three separability and three-party entanglement, we calculated the expectation value of the stabilizer witness $\operatorname{Tr}\left\{W \rho_{\text {exp }}\right\}$, where $W=I^{\otimes 4}-\sigma_{x}^{\otimes 4}-\sigma_{y}^{\otimes 4}-$ $\sigma_{z}^{\otimes 4}$ and $I$ is the two-dimensional identity operator, and $\sigma_{x}, \sigma_{y}$, and $\sigma_{z}$ are the Pauli operators [10], [16]. We obtained $\operatorname{Tr}\left\{W \rho_{\text {exp }}\right\}=-1.51 \pm 0.01$; the values for the ideal Smolin state and the $\rho\left(\psi^{+}\right)$is -2 . Note that no separable, triseparable and one-three separable states (e.g., $\mathrm{A}|\mathrm{B}| \mathrm{C}|\mathrm{D}, \mathrm{A}| \mathrm{B} \mid \mathrm{CD}$, and $\mathrm{A} \mid \mathrm{BCD}$ ) have negative values of the witness. The negative witness value, therefore, indicates that $\rho_{\exp }$ has a considerable amount of entanglement, while it is almost undistillable as indicated by the negativity described above. These analyses clearly demonstrate that the state we generated has bound entanglement.

Finally, it is worth discussing the reason for the degraded fidelity of the Smolin state we prepared. From the reconstructed density matrix of each Bell state we generated, we expected the fidelity of the prepared Smolin state to be 0.980 , assuming perfectly equal statistical mixture of pairs of the four Bell states. Thus, the degraded fidelity of the reconstructed density matrix $\rho_{\text {exp }}$ possibly originates from insufficient statistics of our measurements. To improve the fidelity, and thus the negativities and witness of the prepared Smolin state, longer measurement time with more random and uniform distribution between $\rho\left(\psi^{+}\right), \rho\left(\psi^{-}\right), \rho\left(\phi^{+}\right)$, and $\rho\left(\phi^{-}\right)$would be necessary. 


\section{Conclusion}

We have experimentally demonstrated the efficient generation and characterization of the four-qubit bound Smolin state, encoded in photon-polarization qubits. We reconstructed the density matrix of the genetated state by full quantum state tomography with $\sim 10$ times shorter measurement time than that of the previous demonstration of bound entanglement. The analyses of the reconstructed density matrix showed that the generated state has almost no distillable entanglement between any two parties, while the state still contains a considerable amount of entanglement. These results demonstrate the bound entanglement involved in the generated state. Further experiments to demonstrate QIP protocols using the Smolin state are in progress.

This work was supported by a Grant-in-Aid for Creative Scientific Research (17GS1204) from the Japan Society for the Promotion of Science.

\section{References}

[1] M. A. Nielsen and I. L. Chuang, Quantum Computation and Quantum Information, Cambridge University Press, 2000.

[2] D. Bouwmeester, J.-W. Pan, K. Mattle, M. Eibl., H. Weinfurter, and A. Zeilinger, "Experimental quantum teleportation," Nature, vol.390, pp.575-579, 1997.

[3] P. Walther, K. J. Resch, T. Rudolph, E. Schenck, H. Weinfurter, V. Vedral, M. Aspelmeyer, and a Zeilinger, "Experimental one-way quantum computing," Nature, vol.434, pp.169-176, 2005.

[4] M. Horodecki, P. Horodecki, and R. Horodecki, "Mixed-State Entanglement and Distillation: Is there a 'Bound' Entanglement in Nature?," Phys. Rev. Lett., vol.80, pp.5329-5242, 1998.

[5] J. A. Smolin, "Four-party unlockable bound entangled state," Phys. Rev. A, vol.63, 032306, 2001.

[6] P. Shor, J. Smolin, and A. Thapliyal, "Superactivation of Bound Entanglement," Phys. Rev. Lett., vol.90, 107901, 2003.

[7] M. Murao and V. Vedral, "Remote Information Concentration Using a Bound Entangled State," Phys. Rev. Lett., vol.86, pp.352-355, 2001.

[8] K. Horodecki, M. Horodecki, P. Horodecki, and J. Oppenheim, "Secure Key from Bound Entanglement," Phys. Rev. Lett., vol.94, 160502, 2005.

[9] S. Ishizaka, "Bound Entanglement Provides Convertibility of Pure Entangled States," Phys. Rev. Lett., vol.93, 190501, 2004.

[10] E. Amselem and M. Bourennane, "Experimental four-qubit bound entanglement," Nature Phys., vol.5, pp.748-752, 2009.

[11] J. Lavoie, R. Kaltenbaek, M. Piani, and K. J. Resch, "Experimental bound entanglement in a four-photon state," Phys. Rev. Lett., vol.105, 130501, 2010.
[12] F. Kaneda, R. Shimizu, Y. Mitsumori, H. Kosaka, and K. Edamatsu, "Experimental demonstration of bound entanglement in optical qubits," Proceedings of SOIMGCOE09, pp.21-22, 2009

[13] C. Y. Lu, X. Q. Zhou, O. Gühne, W. B. Gao, J. Zhang, Z. S. Yuan, A. Goebel, T. Yang, and J. W. Pan, "Experimental entanglement of six photons in graph states," Nature Phys., vol.3, pp.91-95, Jan. 2007.

[14] D. F. V. James, P. G. Kwiat, W. J. Munro, and A. G. White, "Measurement of qubits," Phys. Rev. A, vol.64, 052312, 2001.

[15] G. Vidal and R. Werner, "Computable measure of entanglement," Phys. Rev. A, vol.65, 032314, 2002.

[16] G. Tóth and O. Gühne, "Entanglement detection in the stabilizer formalism," Phys. Rev. A, vol.72, 022340, 2005.

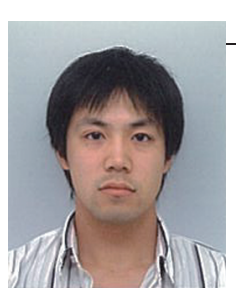

\section{Fumihiro KANEDA}

Fumihiro KANEDA is a Ph.D. student in Department of Electronic Engineering, Graduate School of Engineering, Tohoku University.

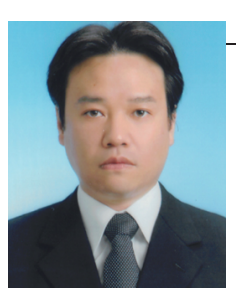

\section{Ryosuke SHIMIZU}

Ryosuke SHIMIZU is an associate professor of Center for Frontier Science and Engineering, University of Electro-Communications, and a researcher at PRESTO, Japan Science and Technology Agency.

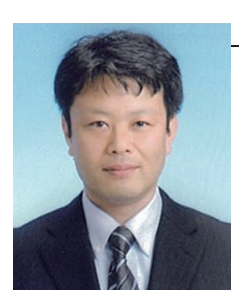

\section{Yasuyoshi MITSUMORI}

Yasuyoshi MITSUMORI is an assistant professor of Research Institute of Electrical Communication, Tohoku University. 


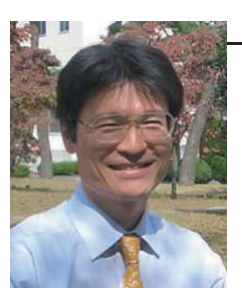

Hiedo KOSAKA

Hiedo KOSAKA is an associate pro-

fessor of Research Institute of Electrical Communication, Tohoku University.

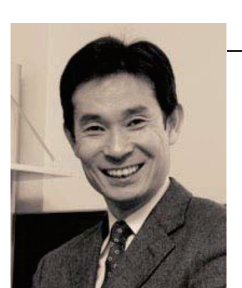

\section{Keiichi EDAMATSU}

Keiichi EDAMATSU is a Professor of Research Institute of Electrical Communication, Tohoku University. He received B.S., M.S., and D.S. degrees in Physics from Tohoku University. He was a Research Associate in Department of Applied Physics, Faculty of Engineering, Tohoku University, a Visiting Associate in Norman Bridge Laboratory of Physics, California Institute of Technology, and an Associate Professor in Division of Materials Physics, Graduate School of Engineering Science, Osaka University. He is a member of the Physical Society of Japan and Optical Society of America. His current research interests are solid-state photophysics, quantum optics, and quantum information/communication science. 